\title{
¿Cuál es el Rol de la Cirugía en el Tratamiento de un Paciente con Cáncer de la Vesícula Biliar Estadio IIIb?
}

\author{
What is the Role of Surgery in the Treatment of a Patient with Stage IIIb Gallbladder Cancer
}

\author{
Carlos Manterola, ${ }^{* * * * * * *} ;$ Roque Conejeros ${ }^{* * * * *} \&$ Eduardo Yáñez ${ }^{* * * *}$
}

MANTEROLA, C.; CONEJEROS, R. \& YÁÑEZ, E. ¿Cuál es el rol de la cirugía en el tratamiento de un paciente con cáncer de la vesícula biliar estadio IIIb? Int. J. Morphol., 33(2):653-659, 2015.

RESUMEN: El cáncer de la vesícula biliar (CaVB), es una neoplasias frecuente en nuestro país. La supervivencia (SV) global no supera el $40 \%$ a 5 años. La invasión de la túnica serosa y el estadio IIIB, se asocian a menor SV por considerarse una etapa avanzada de la enfermedad; por ello no hay consenso respecto del rol de la cirugía en estos casos. El objetivo de este artículo es analizar la evidencia existente respecto del rol de la cirugía en el tratamiento de un paciente con CaVB estadio IIIb. Se realizó una búsqueda sistemática de la evidencia disponible en la bases de datos Clinical Evidence, National Health Service, Health Technology Assessment, Tripdatabase, Cochrane Library y PubMed. Se buscaron a documentos de resumen de la evidencia (overviews, guías de práctica clínica (GPC, tablas GRADE), artículos secundarios (revisiones sistemáticas/RS) y artículos primarios (ensayos clínicos/EC y estudios observacionales/EO). Posteriormente, la evidencia se clasificó según la propuesta de la CEBM 2009. Se encontró un total de 420 documentos relacionados: 25 overviews, 15 tablas GRADE, 30 GPC, 37 RS, 99 EC y 214 EO. Al examinar en detalle todos los documentos; Se comprobó que sólo 17 estaban relacionados con los resultados del tratamiento quirúrgico de la CaVB en etapa III; y 5 se refieren a esta (3 EO, 1 GPC y una recomendación del NCI), pero ninguno al estadio IIIb. Existen pocos estudios relacionados, la mayor parte de ellos son de tipo retrospectivo, con un pequeño número de pacientes incluidos, de población y procedimientos quirúrgicos heterogéneos; por ende, es muy sacar conclusiones y realizar recomendaciones basadas en la evidencia existente.

PALABRAS CLAVE: Neoplasia de la vesícula biliar; Cirugía de la neoplasia de la vesícula biliar; Procedimientos quirúrgicos sobre la vesícula biliar; Cáncer de la vesícula biliar; Cáncer de la vesícula biliar estadio III; Linfadenectomía.

\section{INTRODUCCIÓN}

El cáncer de la vesícula biliar (CaVB), es la una de las neoplasias que más defunciones por causa oncológica genera en las mujeres. La supervivencia (SV) actuarial global de una serie 745 pacientes fue de $38 \%$ a cinco años (Roa et al., 2010, 2014); por ende, se trata de un problema de salud pública por el que deben tomarse decisiones contundentes para dar solución a cada uno de los problemas que involucra.

Sin embargo, la toma de decisiones en salud, se debe basar en conocimientos técnicos y evidencias respecto de las opciones a considerar ante los diversos escenarios que pueden ocurrir respecto de la misma patología. Para ello, se puede recurrir a información previamente generada, como resúmenes de la evidencia, guías de práctica clínica basadas en la evidencia (GPC), artículos secundarios como las revi- siones sistemáticas (RS); y artículos primarios como ensayos clínicos (EC), estudios observacionales (EO), etc.

El objetivo de este artículo es analizar la evidencia existente respecto del rol de la cirugía en el tratamiento de un paciente con CaVB estadio IIIb.

Para ello, se expondrá un caso clínico real, a partir del cual se desarrollará la búsqueda de la evidencia y la discusión posterior.

Se trata de un paciente de sexo femenino, 55 años de edad, residente de la ciudad de Temuco, Región de la Araucanía; con antecedentes de obesidad tipo II e hipertensión arterial esencial en tratamiento. En septiembre de 2014, se le practicó una colecistectomía por colecistitis

* Departamento de Cirugía y Traumatología, Universidad de La Frontera, Temuco, Chile.

** Centro de Excelencia en Estudio Morfológicos y Quirúrgicos (CEMyQ), Universidad de La Frontera, Temuco, Chile.

**** Centro de Investigación en Biociencias, Universidad Autónoma de Chile, Temuco, Chile.

${ }^{* * * * *}$ Instituto Clínico Oncológico del Sur (ICOS), Chile, Temuco, Chile. 
crónica litiásica. La evolución postoperatoria fue favorable, fue dada de alta al segundo día sin inconvenientes y volvió a control a los 10 días de la cirugía, con el informe de su biopsia, que concluyó: "adenocarcinoma poco diferenciado infiltrante hasta serosa". Se le realizó un PET CT el 01/10/ 2014 que permitió verificar una lesión sospechosa de residuo neoplásico a nivel del lecho hepático y un nódulo peritoneal.

Después de una serie de consideraciones y valoraciones clínicas, la paciente fue reintervenida el 11/11/2014, oportunidad en la que se le practicó una cuña hepática y linfadenectomía regional. La paciente evolucionó sin complicaciones, fue dada de alta a su domicilio a los cinco días de su intervención.

Volvió a control clínico dos semanas después con el resultado de la biopsia de la pieza quirúrgica, que concluyó: "linfonodo retrocoledociano con micro metástasis intranodal bien diferenciado. Hígado infiltrado por carcinoma poco diferenciado con márgenes libres. Ganglio peritoneal negativo. Muñón cístico libre de neoplasia. Fragmento de tejido adiposo con inflamación crónica, aguda y fibrosis".

Para ordenar un poco las ideas, hemos de recordar que se trata de un caso con infiltración de la túnica serosa, con un linfonodo retrocoledociano e hígado; por ende, el primer problema es clasificarlo utilizando alguna de las clasificaciones existentes. ¿Cuál de ellas, Nevin V, TNM, UICC, AJCC?

Según la clasificación AJCC, en su $7^{\mathrm{a}}$ edición (National Cancer Institute, 2015); la paciente se encontraba en un estadio IIIb; por ende, con una enfermedad avanzada. Se debe considerar entonces la aplicación del concepto de operabilidad; y si este se acepta, el de resecabilidad. De hecho, el mismo NCI sugiere que a partir de estadio II en adelante, el CaVB sería irresecable, por ende el tratamiento sería sólo paliación (National Cancer Institute).

Como dato al margen, hay evidencia que sugiere que la expectativa de SV de este tipo de pacientes, dejados a su libre evolución es de $0 \%$ a $8 \%$ a 5 años (Ríos Zamudio et al., 2001; ACS, 2015).

A la luz de esta información cabe plantearse la siguiente pregunta: ¿Debió ser reoperada, por tratarse de una enfermedad avanzada?

\section{MATERIAL Y MÉTODO}

Para dar respuesta a las interrogantes antes señaladas, se realizó una búsqueda sistemática de la evidencia dis- ponible, utilizando las siguientes bases de datos: BMJ Clinical Evidence (http://clinicalevidence.bmj.com/x/ index.html), National Health Service (NHS), (http:// www.nhs.uk/Pages/HomePage.aspx), Health Technology Assessment (HTA) (http://www.nets.nihr.ac.uk/programmes/ hta), Tripdatabase (http://www.tripdatabase.com), Cochrane Library (http://www.cochranelibrary.com) (y PubMed (http:/ /www.ncbi.nlm.nih.gov/pubmed).

Se utilizaron los términos MeSH Gallbladder Neoplasms, Gallbladder Neoplasms/surgery, Biliary Tract Surgical Procedures; los términos libres gallbladder cancer, stage III gallbladder cancer, T3 gallbladder cancer, lymph node dissection.

La búsqueda se orientó a documentos de resumen de la evidencia o revisiones globales de la evidencia (overviews), tablas GRADE, GPC, artículos secundarios (RS) y artículos primarios (como EC y EO [estudios de cohortes, estudios comparativos no definidos, series de casos, reportes de casos]).

Posteriormente, la evidencia encontrada se clasificó según la propuesta de la CEBM 2009 (Manterola et al., 2014), resumiéndose de esta forma los hallazgos relacionados al caso clínico en cuestión.

\section{RESULTADOS}

Se puede resumir en las Tablas I y II; en las que se verifica la exigua cantidad de artículos encontrados en estas bases de datos. 25 overviews, 15 tablas GRADE, 30 GPC, 37 RS, 99 EC, y 214 EO; inicialmente relacionadas con CaVB.

$\mathrm{Al}$ revisar en extenso todos los documentos antes señalados $(n=420)$; se pudo verificar que sólo 17 se relacionaban con resultados del tratamiento quirúrgico del $\mathrm{CaVB}$, en que se mencionaba el estadio III 8 (1 GPC, 1 EC, 2 RS, 1 revisión narrativa o no sistemática; y 12 EO, de los cuales 6 corresponden a SC); los que al clasificar por niveles de evidencia, se resumen en la Tabla III por tipo de diseño y nivel de evidencia que aportan.

De estos, se encontró evidencia directa asociada a el rol de la cirugía (colecistectomía extendida) en el estadio III del CaVB en 5 documentos (Ausania et al., 2013; Choi et al., 2013; Goetze \& Paolucci, 2014; Lee et al., 2014, NCI), a pesar que algunos sólo lo tocan de forma tangencial. Sin embargo, ninguno de ellos, se refiere a estadio IIIb. El aporte de estos, se resume en la Tabla IV. 
Tabla I. Artículos encontrados, relacionados con CaVB, diseños y niveles de evidencia.

\begin{tabular}{lccc}
\hline Fuentes & $\begin{array}{c}\text { Systematic } \\
\text { Overviews }\end{array}$ & $\begin{array}{c}\text { Tablas } \\
\text { GRADE }\end{array}$ & $\begin{array}{c}\text { Guías de } \\
\text { práctica clínica }\end{array}$ \\
\hline BMJ Clinical Evidence & $24 / 0$ & $15 / 0$ & $29 / 0$ \\
NHS / UK & 0 & 0 & 0 \\
HTA / UK & 0 & 0 & 0 \\
Tripdatabase & 0 & 0 & 0 \\
Cochrane Library & $1 / 0$ & 0 & 0 \\
PubMed & 0 & 0 & $1 / 1$ \\
Total & $\mathbf{2 5 / 0}$ & $\mathbf{1 5 / 0}$ & $\mathbf{3 0} / \mathbf{1}$ \\
\hline
\end{tabular}

Tabla II. Artículos encontrados, relacionados con CaVB, diseños y niveles de evidencia.

\begin{tabular}{lccc}
\hline Fuentes & RS & EC & EO \\
\hline BMJ Clinical Evidence & 0 & 0 & 0 \\
NHS / UK & 0 & $5 / 0$ & $137 / 0$ \\
HTA / UK & 0 & 0 & 0 \\
Tripdatabase & 0 & 0 & $4 / 4$ \\
Cochra ne Library & $10 / 0$ & $62 / 0$ & $20 / 2$ \\
PubMed & $27 / 3$ & $33 / 1$ & $52 / 6$ \\
Total & $37 / 3$ & $100 / 2$ & $213 / 11$ \\
\hline
\end{tabular}

$\mathrm{RS}=$ Revisión sistemática, $\mathrm{EC}=$ Ensayo clínico, EO= Estudios observacionales (incluye series de casos, cohortes y estudios comparativos).

Tabla III. Artículos encontrados relacionados con cirugía del CaVB, diseños y niveles de evidencia.

\begin{tabular}{|c|c|c|c|}
\hline Autores & Temática del artículo & Diseño & NE \\
\hline Agarwal et al. (2013) & Rol de la estadificación laparoscópica en CaVB. & $\mathrm{EC}(n=409)$ & $2 b$ \\
\hline Ausania et al. (2013) & $\begin{array}{l}\text { Resultados de la estadificación a } 3 \text { meses antes de una resección radical } \\
\text { en pacientes con CaVB incidental post colecistectomía. }\end{array}$ & EO $\quad(n=49)$ & 4 \\
\hline Ballo et al. (2015) & $\begin{array}{l}\text { Influencia de la colecistectomía laparoscópica en el resultado de la } \\
\text { resección oncológica de un CaVB. }\end{array}$ & EO $\quad(n=20)$ & $2 b$ \\
\hline Birnbaum et al. (2015) & Linfadenectomía en CaVB. Extensión y v alor pronóstico de la & $\mathrm{SC} \quad(\mathrm{n}=112)$ & 4 \\
\hline Choi et al. (2013) & $\begin{array}{l}\text { nronorción de nndos linfáticns rese cad s } \\
\text { Necesidad de resección de la vía biliar de forma rutinaria como } \\
\text { estrategia quirúr gica para pacientes con CaVB T2 v T3. }\end{array}$ & EO $(n=71)$ & 4 \\
\hline Coburn et al. (2008) & Cirugía del CaVB. Análisis de un estudio poblacional. & $\mathrm{SC}(n=150)$ & 4 \\
\hline Goetze \& Paolucci (2014) & Resultados del registro alemán de colecistectomía extendida como & $\mathrm{EO}(\mathrm{n}=283)$ & 4 \\
\hline Gumbs \& Hoffman (2010) & $\begin{array}{l}\text { factor pronóstico en CaVB incidentales T1b-T3. } \\
\text { Colecistectomía radical laparoscópica en pacientes con CaVB T2. }\end{array}$ & $\operatorname{RS}(n=3)$ & 4 \\
\hline Is ambert et al. (2011) & $\begin{array}{l}\text { Cuándo, por qué y que operación se debe realizar en CaVB descubierto } \\
\text { incidentalmente? }\end{array}$ & $\mathrm{RN} \quad(\mathrm{n}=0)$ & $3 a$ \\
\hline Ito et al. (2004) & Resultados de la cirugía del CaVB. Veinte años de experiencia. & $\mathrm{SC} \quad(n=48)$ & 4 \\
\hline Jeong et al., 2013 & Colecistectomía laparoscópica radical para CaVB T2. & $\mathrm{SC} \quad(n=45)$ & 4 \\
\hline Lee et al. (2012b) & Estrate gia quirúrgica para CaVB T2: estudio multicéntrico. & EO $\quad(n=258)$ & 4 \\
\hline Lee et al. (2011) & Tratamiento quirúrgico del CaVB T1. & RS $(n=29$ AP $)$ & $3 \mathrm{a}$ \\
\hline Lee et al. (2014) & GPC de la asociación coreana de cirugía hepatobiliar y pancreática para & GPC & ---- \\
\hline Shindoh et al. (2015) & $\begin{array}{l}\text { La localización del tumor como predictor de progresión tumoral y SV } \\
\text { en pacientes con } \mathrm{CaVB} \mathrm{T} 2 \text {. }\end{array}$ & EO $\quad(n=252)$ & 4 \\
\hline Shukla \& Barreto (2010) & Se debe resecar rutinariamente la vía biliar extrahepática en CaVB? & $\operatorname{RS}(n=5$ AP $)$ & $3 \mathrm{a}$ \\
\hline Wakabayashi et al. (2004) & Factores pronósticos postoperatorios en estadios III y IV de CaVB. & $\mathrm{SC} \quad(n=37)$ & 4 \\
\hline
\end{tabular}

$\mathrm{AP}=$ Artículos primarios, $\mathrm{NE}=$ Nivel de evidencia, $\mathrm{GPC}=$ Guía de práctica clínica, $\mathrm{EC}=$ Ensayo clínico, $\mathrm{EO}=\mathrm{Estudio}$ observacional, $\mathrm{SC}=\mathrm{Serie}$ de casos, $\mathrm{RC}=$ Reporte de casos, $\mathrm{RN}=$ Revisión narrativa. 
Tabla IV. Artículos relacionados con cirugía del CaVB estadio III.

\begin{tabular}{|c|c|}
\hline $\begin{array}{l}\text { Autores } \\
\text { Diseño }\end{array}$ & A poya rol de la cirugía? \\
\hline Ausania et al. (2013) & Sí, pero después de realizar estadificación preoperatoria con TAC y laparoscopia. \\
\hline $\mathrm{EO}(\mathrm{n}=49)$ & En los resecados se observó mejor SV que en los no resecados. \\
\hline $\begin{array}{l}\text { Choi et al., } 2013 . \\
\text { EO }(\mathrm{n}=71)\end{array}$ & $\begin{array}{l}\text { Sí. La SV es mejor que en los pacientes resecados. Sin embargo, la resección de } \\
\text { la vía biliar de forma rutinaria no sería necesaria como parte de la estrategia } \\
\text { quirúrgica para pacientes con CaVB T2 y T3. }\end{array}$ \\
\hline $\begin{array}{l}\text { Goetze \& Paolucci (2014) } \\
\text { EO }(\mathrm{N}=283)\end{array}$ & $\begin{array}{l}\text { Sí. L a SV fue mejor en pacientes T3 resecados }(n=75) \text { que en a quellos no } \\
\text { resecados }(n=207) \text {. Sin embargo, se requiere de estrategias más agresivas que en } \\
\text { T1b y } 2 \text {. }\end{array}$ \\
\hline $\begin{array}{l}\text { Lee et al. (2014) } \\
\text { GPC }\end{array}$ & Sí si se trata de un paciente resecable. Sugieren adyuvancia postoperatoria. \\
\hline NCI (2015) & $\begin{array}{l}\text { En la información por estadios para CaVB, la NCI NO recomienda la cirugía } \\
\text { para estadio III, por considerarla una enfermedad avanzada. }\end{array}$ \\
\hline
\end{tabular}

\section{DISCUSIÓN}

Es complejo tomar decisiones en salud cuando existe poca o nula evidencia; o la existente es de un pobre nivel de evidencia y basada en su mayoría en series de casos de carácter retrospectivo y de pequeño número de pacientes.

Sin embargo, se ha de comentar algunos aspectos respecto de los cuales existe alguna evidencia: uno de ello dice relación con los factores pronósticos del CaVB. Entre ellos destacan el nivel de infiltración de la pared vesicular, la existencia de infiltración linfática, el grado de diferenciación; así como la infiltración perineural y las permeaciones vasculares (Roa et al., 2014); la localización hepática respecto de la peritoneal (Shindoh et al., 2015); y el compromiso de los senos de Rokitanski Aschoff (SRA) (Fig. 1) (Roa et al., 2013). Del mismo modo, existe evidencia en relación a que la colesterolosis actúa como factor protector del desarrollo de CaVB (9,2 veces menos riesgo el tenerla que el no tenerla) (Roa et al., 2010).

Por ende, y respecto de la escasa evidencia encontrada, la pregunta parece razonable contestarla con el algoritmo que hemos venido utilizando desde hace algunos años (Fig. 2). Colecistectomía y observación rutinaria para CaVB con infiltración hasta la mucosa; colecistectomía extendida para CaVB con infiltración hasta las túnicas muscular (T1b), subserosa (T2), serosa (T3) y más allá de la serosa (T4). Señalando además que la resección hepática, realizamos una resección segmentaria que incluye los segmentos IVb y V; y la linfadenectomía que realizamos, corresponde a la extirpación de todo el tejido linfograso de los territorios N1-N2; intentando como meta, la exéresis de un número cercano a 10 linfonodos. En pacientes con T3 y T4 se evalúa caso a caso la indicación de cirugía. De este modo; obtuvimos SV actuarial a 5 años de $39 \%$ y $12,5 \%$ para CaVB T3 y T4 respectivamente; aún cuando la mediana real de SV para ambos fue de 14,5 y 5,5 meses respectivamente (Manterola et al., 2010).

Sin embargo, una parte fundamental en nuestro algoritmo diagnóstico terapéutico, es el acucioso estudio histopatológico de la pieza de colecistectomía; la que se concluye con el denominado "mapeo vesicular" (Fig. 3); puesto que hemos verificado que tiene implicancia no solo operacional sino también de tipo pronóstico para los pacientes.

Respecto de la magnitud de la resección hepática; existe evidencia que apoya la simple resección de lecho hepático (resección de margen de $2 \mathrm{~cm}$ de parénquima en el lecho vesicular), hasta la hepatectomía derecha extendida, pasando por segmentectomía IVb-V y segmentectomía IVbV-VI. No parece haber relación directa entre la magnitud de la resección hepática y la SV (Lee et al., 2014).

Por otra parte, a pesar de lo avanzado, aún existen controversias terapéuticas en esta materia. Entre las que destacan la indicación de la colecistectomía extendida en tumores pT1a y pT1b1 y asociado a esto, el rol de los SRA (Roa et al., 2013); el rol de la colecistectomía extendida en pacientes con invasión de la serosa o más allá de ésta (de Aretxabala, et al., 2009; Manterola et al., 2010); la utilidad de la quimio radioterapia (de Aretxabala, et al., 1999; de Aretxabala, et al., 2009; Lee et al., 2012a); etc.

Respecto del primer punto señalado en el párrafo anterior, hay un artículo de reciente publicación, en el que se verificó que la SV de pacientes con pT1a (infiltración mucosa) y pT1b (infiltración muscular) fue de $87,6 \%$ y 


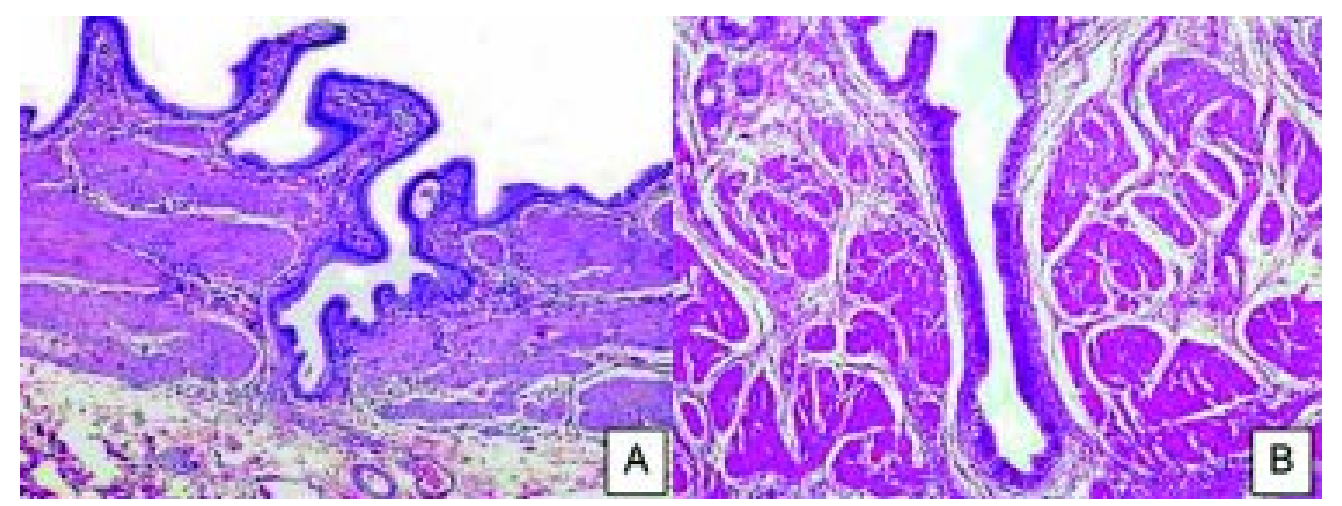

Fig. 1. Senos de Rokitansky Aschoff.

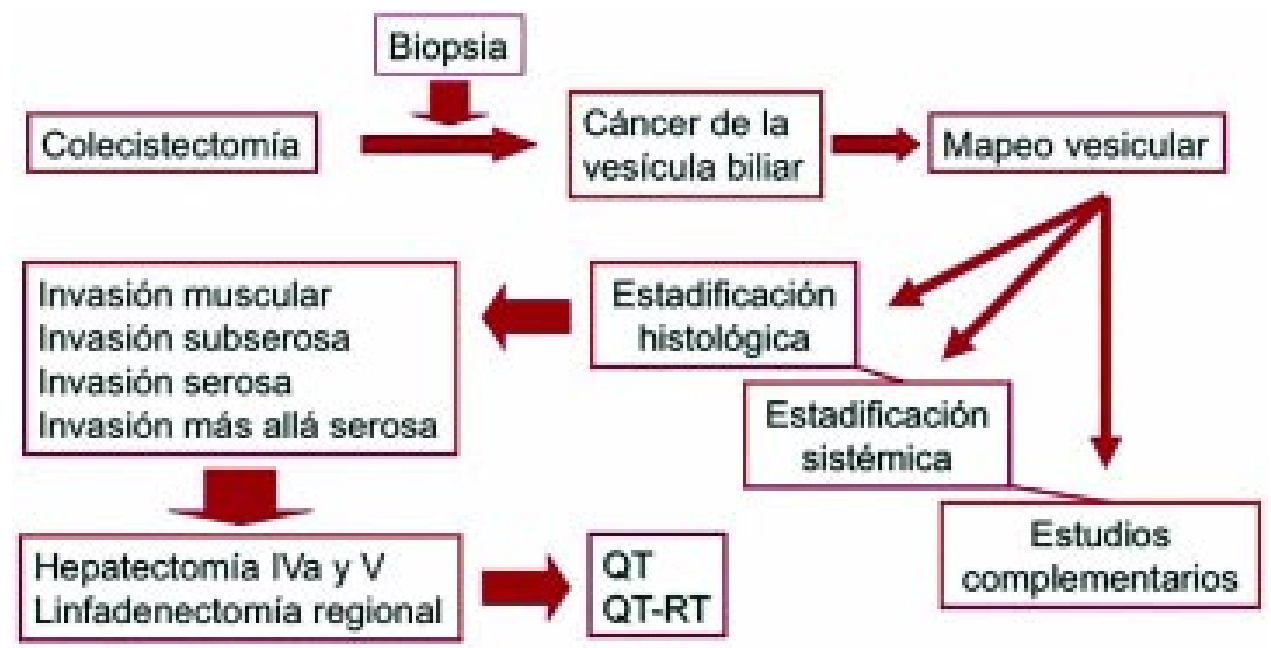

Fig. 2. Algoritmo diagnostico terapéutico en pacientes con CaVB que se diagnostica en la pieza quirúrgica de la colecistectomía.

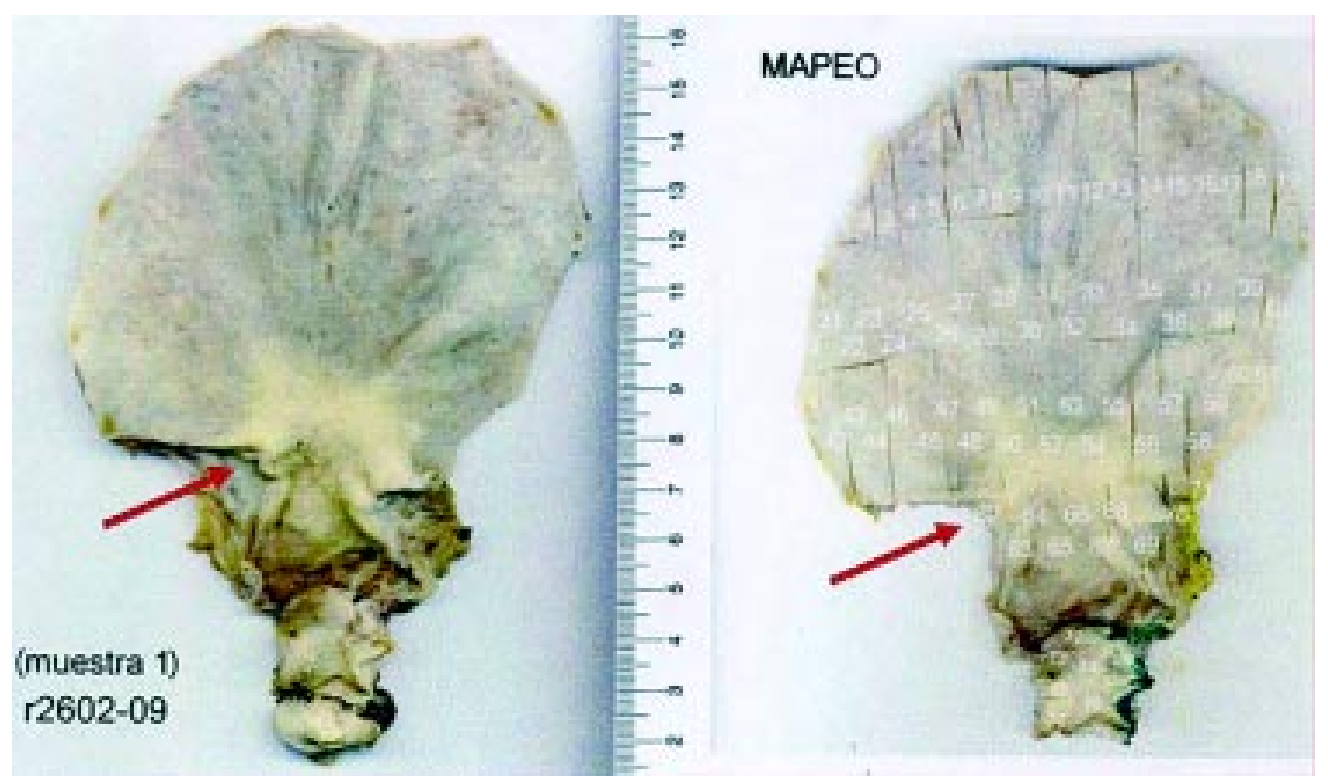

Fig. 3. Mapeo de una pieza de colecistectomía. Adenocarcinoma tubular moderadamente diferenciado, infiltrante hasta la serosa. Metástasis ganglionar linfática. Pared biliar infiltrada por adenocarcinoma con permeaciones tumorales perineurales 
$86,4 \%$, respectivamente al ser tratados con colecistectomía. Sin embargo, pacientes con RAS (+) presentaron SV hasta 30 puntos porcentuales inferiores (Roa et al., 2013).

En referencia a algunas novedades terapéuticas, se han publicado algunos artículos cuyos resultados parecen alentadores. Uno de ellos, es un estudio coreano, fase III; en el que se demuestra la eficacia del esquema asociado con gemcitabina, oxaliplatino y erlotinib (Yang et al., 2013). Otro, es un estudio Fase II con Gemcitabina, cisplatino, oxaliplatino y 5-FU (Hezel et al., 2010).

Otro hecho de interés son los estudios asociados a la genética del cáncer vesicular; entre los que destaca la evidencia de las frecuentes mutaciones de genes y oncogenes. Por ejemplo, del oncogen KRAS (19\% a 38\%); del gen supresor TP53 (36\%); del oncogen BRAF (33\%); y del gen supresor P16INK4A (31\% a 62\%). A estos apuntarán poste- riormente las denominadas "Targeted therapies" (Hezel et al.; Zhu \& Hezel, 2011; Marino et al., 2013).

Pero, volviendo a las interrogantes que planteamos intentar aclarar a la luz de la evidencia actual, podemos señalar lo siguiente: en relación a la indicación de reintervención nos parece que la respuesta es sí, con base en: nuestra propia experiencia (Manterola et al., 2010); una GPC de origen coreano (Lee et al., 2014); y tres EO (Ausania et al., 2013; Choi et al.; Goetze \& Paolucci). Es decir con base en un nivel de evidencia 4 .

En resumen, gran parte de los pocos estudios recolectados son de tipo retrospectivos, con un pequeño número de pacientes incluidos; heterogeneidad de poblaciones y procedimientos quirúrgicos. Por lo tanto, es muy difícil poder sacar conclusiones y realizar recomendaciones basadas en la evidencia existente.

MANTEROLA, C.; CONEJEROS, R. \& YÁÑEZ, E. What is the role of surgery in the treatment of a patient with stage IIIb gallbladder cancer? Int. J. Morphol., 33(2):653-659, 2015.

SUMMARY: Gallbladder cancer (GBC), is a common neoplasm in our country. The overall survival rate (OSR) does not exceed $40 \%$ at 5 years. The invasion of the serosa and IIIB stage, are associated with lower OSR seen it are an advanced stage of the disease, so there is no consensus on the role of surgery in this type ofpatients. The aim of this study is to analyze the existing evidence concerning the role of surgery in the treatment of a patient with stage IIIb GBC. A systematic search of available evidence in the databases Clinical Evidence, National Health Service, Health Technology Assessment, Tripdatabase, Cochrane Library and PubMed search was performed. Evidence summary documents (overviews, GRADE tables, Clinical Guidelines/CG), secondary articles (systematic reviews) and primary articles (Clinical trials/CT) and observational studies/OS) were searched. Subsequently, evidence was classified as proposed by EMBC 2009. A total of 420 related documents were found: 25 overviews, 15 GRADE tables, 30 CG, 37 SR, 99 CT and 214 OS. In reviewing at length all documents; It was verified that only 17 were related to results of surgical treatment of GC that stage III was mentioned and 5 refer to this ( $3 \mathrm{OS}, 1 \mathrm{CG}$ and a recommendation from NCI), but none of them to the IIIb stage. There are few related studies, most of them are retrospective, with a small number of patients included, heterogeneous population and surgical procedures; thereby, it is difficult to draw conclusions and make recommendations based on the evidence.

KEY WORDS: Gallbladder Neoplasm; Gallbladder Neoplasms/surgery; Biliary Tract Surgical Procedures; Gallbladder cancer; Stage III gallbladder cancer; Lymph node dissection.

\section{REFERENCIAS BIBLIOGRÁFICAS}

Agarwal, A. K.; Kalayarasan, R.; Javed, A.; Gupta, N. \& Nag, H. H. The role of staging laparoscopy in primary gall bladder cancer--an analysis of 409 patients: a prospective study to evaluate the role of staging laparoscopy in the management of gallbladder cancer. Ann. Surg.,258(2):318-23, 2013.

American Cancer Society (ACS). Estadísticas de supervivencia para el cáncer de vesícula biliar, según la etapa. 2015. Disponible enhttp:// www.cancer.org/espanol/cancer/cancer devesiculabiliar/guiadetallada/ cancer-de-vesicula-biliar-early-survival-rates

Ausania, F.; Tsirlis, T.; White, S. A.; French, J. J.; Jaques, B. C.; Charnley, R. M. \& Manas, D. M. Incidental pT2-T3 gallbladder cancer after a cholecystectomy: outcome of staging at 3 months prior to a radical resection. H. P. B. (Oxford), 15(8):633-7, 2013.
Ballo, R.; Saeed, M.; Daly, S.; Pinzon, M.; Francescatti, A.; Millikan, K. W.; Myers, J. A.; Bines, S. F. \& Luu, M. B. Does initial laparoscopic cholecystectomy influence the outcomes of definitive oncologic resection for gallbladder cancer? Am. Surg., 81(2):E54-6, 2015.

Birnbaum, D. J.; Viganò, L.; Russolillo, N.; Langella, S.; Ferrero, A. \& Capussotti, L. Lymph node metastases in patients undergoing surgery for a gallbladder cancer. Extension of the lymph node dissection and prognostic value of the lymph node ratio. Ann. Surg. Oncol., 22(3):8118, 2015.

Choi, S. B.; Han, H. J.; Kim, W. B.; Song, T. J.; Suh, S. O. \& Choi, S. Y. Surgical strategy for T2 and T3 gallbladder cancer: is extrahepatic bile duct resection always necessary? Langenbecks Arch. Surg., 398(8):1137-44, 2013. 
Coburn, N. G.; Cleary, S. P.; Tan, J. C. \& Law, C. H. Surgery for gallbladder cancer: a population-based analysis. J. Am. Coll. Surg., 207(3):37182, 2008.

de Aretxabala, X.; Roa, I.; Burgos, L.; Cartes, R.; Silva, J.; Yañez, E.; Araya, J. C.; Villaseca, M.; Quijada, I. \& Vittini, C. Preoperative chemoradiotherapy in the treatment of gallbladder cancer. Am. Surg., 65(3):241-6, 1999.

de Aretxabala, X.; Roa, I.; Hepp, J.; Maluenda, F.; Mordojovich, G.; Leon, J. \& Roa, J. C. Early gallbladder cancer: is further treatment necessary? J. Surg. Oncol., 100(7):589-93, 2009.

Goetze, T. O. \& Paolucci, V. Incidental T1b-T3 gallbladder carcinoma. Extended cholecystectomy as an underestimated prognostic factorresults of the German registry. Chirurg., 85(2):131-8, 2014

Gumbs, A. A. \& Hoffman, J. P. Laparoscopic completion radical cholecystectomy for T2 gallbladder cancer. Surg. Endosc., 24(12):3221-3, 2010.

Hezel, A. F.; Deshpande, V. \& Zhu, A. X. Genetics of biliary tract cancers and emerging targeted therapies. J. Clin. Oncol., 28(21):3531-40, 2010.

Isambert, M.; Leux, C.; Métairie, S. \& Paineau, J. Incidentally-discovered gallbladder cancer: When, why and which reoperation? J. Visc. Surg., 148(2):e77-84, 2011.

Ito, H.; Matros, E.; Brooks, D. C.; Osteen, R. T.; Zinner, M. J.; Swanson, R. S.; Ashley, S. W. \& Whang, E. E. Treatment outcomes associated with surgery for gallbladder cancer: a 20 -year experience. $J$. Gastrointest. Surg., 8(2):183-90, 2004.

Jeong, C. Y.; Cho, J. K.; Ju, Y. T.; Jung, E. J.; Lee, Y. J.; Choi, S. K.; Ha, W. S.; Park, S. T. \& Cha, S. Laparoscopic radical cholecystectomy for T2 gallbladder cancer. HPB, 15(Suppl. 2):215, 2013.

Lee, S. E.; Jang, J. Y.; Lim, C. S.; Kang, M. J. \& Kim, S. W. Systematic review on the surgical treatment for T1 gallbladder cancer. World $J$. Gastroenterol., 17(2):174-80, 2011.

Lee, J.; Park, S. H.; Chang, H. M.; Kim, J. S.; Choi, H. J.; Lee, M. A.; Jang, J. S.; Jeung, H. C.; Kang, J. H.; Lee, H. W.; Shin, D. B.; Kang, H. J.; Sun, J. M.; Park, J. O.; Park, Y. S.; Kang, W. K. \& Lim, H. Y. Gemcitabine and oxaliplatin with or without erlotinib in advanced biliary-tract cancer: a multicentre, open-label, randomised, phase 3 study. Lancet Oncol., 13(2):181-8, 2012a.

Lee, S. E.; Jang, J. Y. \& Kim, S. W. Surgical strategy of T2 gallbladder cancer: A multicenter survey. HPB, 14(Suppl. 2):129, 2012 b.

Lee, S. E.; Kim, K. S.; Kim, W. B.; Kim, I. G.; Nah, Y. W.; Ryu, D. H.; Park, J. S.; Yoon, M. H.; Cho, J. Y.; Hong, T. H.; Hwang, D. W.; Choi, D. W. \& Korean Association of Hepato-Biliary and Pancreas Surgery. Practical guidelines for the surgical treatment of gallbladder cancer. $J$. Korean Med. Sci., 29(10):1333-40, 2014.

Manterola, C.; Vial, M. \& Roa, J. C. Survival of a cohort of patients with intermediate and advanced gall bladder cancer treated with a prospective therapeutic protocol. Acta Cir. Bras., 25(3):225-30, 2010.

Manterola, C.; Asenjo-Lobos, C. \& Otzen, T. Jerarquización de la evidencia. Niveles de evidencia y grados de recomendación de uso actual. Rev. Chil. Infectol., 31(6):705-18, 2014.

Marino, D.; Leone, F.; Cavalloni, G.; Cagnazzo, C. \& Aglietta, M. Biliary tract carcinomas: from chemotherapy to targeted therapy. Crit. Rev. Oncol. Hematol., 85(2):136-48, 2013.

National Cancer Institute. Gallbladder Cancer Treatment-for health professionals $\left(\mathrm{PDQ}{ }^{\circledR}\right)$. Stage Information for Gallbladder Cancer. 2015. Disponible en http://www.cancer.gov/cancertopics/pdq/ treatment/gallbladder/HealthProfessional/page3

Ríos Zambudio, A.; Sánchez Bueno, F.; García Marcilla, J. A.; Robles Campos, R.; Tamayo Rodríguez, M. E.; Balsalobre Salmerón, M. D.; Parrilla Paricio, P. Factores pronósticos del cáncer de vesícula. Gastroenterol. Hepatol., 24(6):281-6, 2001.

Roa, I.; Ibacache, G.; Muñoz, S. \& de Aretxabala, X. Gallbladder cancer in Chile: Pathologic characteristics of survival and prognostic factors: analysis of 1,366 cases. Am. J. Clin. Pathol., 141(5):675-82, 2014.

Roa, I.; de Aretxabala, X.; Ibacache, G. \& Muñoz, S. Association between cholesterolosis and gallbladder cancer. Rev. Med. Chil., 138(7):804-8, 2010.

Roa, J. C.; Tapia, O.; Manterola, C.; Villaseca, M.; Guzman, P.; Araya, J. C.; Bagci, P.; Saka, B. \& Adsay, V. Early gallbladder carcinoma has a favorable outcome but Rokitansky-Aschoff sinus involvement is an adverse prognostic factor. Virchows Arch., 463(5):651-61, 2013.

Shindoh, J.; de Aretxabala, X.; Aloia, T. A.; Roa, J. C.; Roa, I.; Zimmitti, G.; Javle, M.; Conrad, C.; Maru, D. M.; Aoki, T.; Vigano, L.; Ribero, D.; Kokudo, N.; Capussotti, L. \& Vauthey, J. N. Tumor location is a strong predictor of tumor progression and survival in T2 gallbladder cancer: an international multicenter study. Ann. Surg., 261(4):733-9, 2015.

Shukla, P. J. \& Barreto, S. G. Systematic review: should routine resection of the extra-hepatic bile duct be performed in gallbladder cancer? Saudi J. Gastroenterol., 16(3):161-7, 2010.

Wakabayashi, H.; Ishimura, K.; Hashimoto, N.; Otani, T.; Kondo, A. \& Maeta, H. Analysis of prognostic factors after surgery for stage III and IV gallbladder cancer. Eur. J. Surg. Oncol., 30(8):842-6, 2004.

Yang, R.; Wang, B.; Chen, Y. J.; Li, H. B.; Hu, J. B. \& Zou, S. Q. Efficacy of gemcitabine plus platinum agents for biliary tract cancers: a metaanalysis. Anticancer Drugs, 24(8):871-7, 2013.

Zhu, A. X. \& Hezel, A. F. Development of molecularly targeted therapies in biliary tract cancers: reassessing the challenges and opportunities. Hepatology, 53(2):695-704, 2011.

\author{
Dirección para Correspondencia: \\ Dr. Carlos Manterola \\ Departamento de Cirugía y Traumatología \\ Universidad de La Frontera \\ Casilla 54-D \\ Temuco \\ CHILE
}

Email: carlos.manterola@ufrontera.cl

Recibido: 10-01-2015

Aceptado: 27-03-2015 